Revue européenne des sciences sociales

European Journal of Social Sciences

XXXVIII-119|2000

La peur de l'impensable dans les sciences sociales

\title{
Faillible droit
}

Olivier Jouanjan

\section{OpenEdition}

\section{Journals}

Édition électronique

URL : http://journals.openedition.org/ress/672

DOI : $10.4000 /$ ress.672

ISSN : 1663-4446

\section{Éditeur}

Librairie Droz

Édition imprimée

Date de publication : 1 décembre 2000

Pagination : 65-78

ISBN : 2-600-00481-5

ISSN : 0048-8046

Référence électronique

Olivier Jouanjan, «Faillible droit », Revue européenne des sciences sociales [En ligne],

XXXVIII-119 | 2000, mis en ligne le 15 décembre 2009, consulté le 04 mai 2019. URL : http://

journals.openedition.org/ress/672; DOI : 10.4000/ress.672 


\section{Olivier JOUANJAN}

\section{FAILLIBLE DROIT}

A première vue, le juriste n'a peur de rien. Précisons : il n'a peur de rien en tant que juriste. Du moins, dans un Etat qu'on peut qualifier d'Etat de droit démocratique, et sous la réserve de n'être pas confronté à un cas extraordinaire qui pourrait, même dans un Etat démocratique et libéral, se révéler être dangereux. On dira donc que, dans un tel système politique - c'est une condition sans doute évidente mais essentielle qu'il faut un système où soit garantis l'indépendance des juges et, à titre de droits effectifs, la liberté de la science, de la recherche et de l'enseignement et les droits de la défense -, dans la pratique ordinaire de son métier, le juriste ne devrait pas nourrir de craintes particulières.

C'est que, à première vue, son activité de juriste - de professeur, de juge, d'avocat, etc. - se déroule précisément dans un monde réglé. L'un des principes structurants de ce monde de règles n'est-il pas, précisément, le principe de la sécurité juridique qui garantit la stabilité et la prévisibilité nécessaires et suffisantes du droit applicable aux situations concrètes. A première vue, ce monde où évolue le juriste ne saurait le prendre par surprise.

De plus, la doctrine classique admet un principe de complétude juridique qui prévient le droit du vide: l'absence d'une règle de niveau déterminé se comble par le recours à un principe plus général de niveau plus élevé. Le droit, comme la nature a horreur du vide. Il n'y a donc pas à s'effrayer d'un silence éternel d'espaces infinis : rien qui donne le vertige. Ce monde ne doit pas prendre le juriste au dépourvu.

Enfin, cet ensemble complet de normes est un ensemble ordonné; il forme système. C'est bien un «monde », et pas un simple agrégat. Les techniques sont nombreuses et assez éprouvées qui permettent de surmonter la contradiction apparente entre règles de droit, de régler ce qu'on appelle les conflits de lois : règles de solution des conflits de lois dans le temps, principe de la hiérarchie des normes, principe de préférence de la règle spéciale par rapport à la règle générale, la panoplie paraît bien fournie ${ }^{1}$ pour éviter au juriste d'être pris au piège de la contradiction.

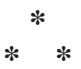

A première vue, donc, le juriste n'a pas de raison de craindre d'être pris en défaut par l'ordre des règles qu'il mobilise afin d'apporter au cas la décision qui

\footnotetext{
1 Pas complète cependant: quel choix faire entre la règle spéciale antérieure et la règle générale pos-
} térieure de même rang? 
lui convient, la décision du cas formant, sans doute, l'intérêt de connaissance de la discipline juridique ${ }^{2}$. Il paraît disposer des principes et techniques nécessaires à la justification des décisions, nécessaires pour, comme l'on dit, fonder la décision en droit. «Fonder la décision»: l'expression emporte avec elle une certaine représentation du travail juridique, une certaine image de l'activité juridique décisionnelle. La concrétisation des règles de droit qu'exige le cas posé s'exécute en tant qu'application du droit à une situation de fait. Il existe une (ou des) norme(s) juridique(s) applicable(s), dormantes pour ainsi dire et qu'il suffirait de réveiller dès que se présente un cas pertinent. Le travail du juriste, outre l'interprétation des règles éventuellement nécessaire pour préparer, affûter les normes en vue de leur application consisterait, guetteur ou rabatteur, à rapporter le cas à la norme, pour le faire tomber sous la règle, dans une sorte de «piège logique» (F. Müller). La forme de l'opération de décision est ainsi syllogistique. La norme (interprétée en tant que de besoin) donne la majeure, le cas fait la mineure, la décision s'identifie à la conclusion. A cette forme de l'opération décisionnelle correspond la structure formelle de la norme juridique, telle que traditionnellement présentée: une proposition hypothétique dont l'antécédent est formé par ce qu'on appelle la «condition» juridique, le conséquent, par la «conséquence de droit». Ainsi le célèbre article 1382 du Code civil ainsi rédigé: «Tout fait quelconque de l'homme, qui cause à autrui un dommage, oblige celui par la faute duquel il est arrivé, à le réparer» peut être récrit de la manière suivante: Si X commet une faute, si Y subit un dommage, si la faute de $\mathrm{X}$ est la cause du dommage subi par Y, X doit réparer le dommage subi par Y. Une série de conditions (l'existence d'un délit imputable, l'existence d'un dommage effectif, l'existence d'un lien de causalité entre le délit et le dommage) détermine une conséquence de droit: l'obligation de réparer. L'application de l'article 1382 du Code civil consiste donc à vérifier que les conditions sont ou non réunies en l'espèce pour tirer la conséquence de droit.

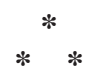

Bien sûr un tel schéma de l'application comme «subsomption » est trop simple et trop beau pour être vrai. Toutefois, on est surpris de constater que même lorsque la réflexion méthodologique se fait plus sophistiquée - et les tentatives sont nombreuses, depuis le début du $\mathrm{XX}^{\mathrm{e}}$ siècle pour compliquer ou relativiser ce modèle du juriste comme «Paragraphenautomat» (Max Weber) ou «bouche de la loi» (Montesquieu) - il s'agit le plus souvent d'environner le modèle formel syllogistique ou de lui plaquer des considérations adjacentes ou adjonctives, d'ornementer la forme logique d'éléments qui viennent en alourdir l'architecture sans toutefois en abandonner l'architectonique.

Ainsi, chez l'auteur d'un des traités les plus importants, en langue allemande tout au moins, de méthodologie juridique, Karl Larenz. Il appartient, selon lui, à la méthodologie du droit de «montrer clairement que l'application du droit ne peut être séparée de l'interprétation ni du perfectionnement jurisprudentiel du droit (Rechtsfortbildung), qu'elle ne repose que pour une petite part sur la sub-

F. Müller, Discours de la méthode juridique, trad. fr. O. Jouanjan, Paris, P.U.F., 1996, p. 214. 
somption logique, que le recours à une pensée de type axiologique est indispensable en droit, qui ne doit toutefois pas être confondue avec l'usage de valeurs personnelles, et de montrer enfin quelles sont les formes intellectuelles - types, critères nécessitant une concrétisation, concept fonctionnel - dont se sert une telle pensée $^{3}$ ». Toutefois, le «schéma logique de l'application de la loi » reste celui du syllogisme ${ }^{4}$. Ce qui n'est pas là remis en cause c'est, aussi sophistiquée qu'elle puisse être, l'application, l'idée d'application du droit, même si on l'affuble parfois, comme chez Larenz, de guillemets.

On applique quelque chose à une autre chose. Le mot application renvoie à un rapport qui s'établit entre deux choses existant normalement séparément mais dont l'une va servir de mesure à l'autre. Ici, la norme d'un côté, les faits de l'autre. Le mot application a pour connotation l'idée selon laquelle les éléments qui participent à l'application, ce qui est appliqué comme ce à quoi cela est appliqué, préexistent séparément. Pour nous juristes, la connotation du langage de l'application, c'est la vision positiviste selon laquelle la norme est dans le texte de la loi et «s'applique» aux «faits» de l'espèce. L'herméneutique d'un Larenz ne remet pas en cause ce schéma et cet héritage-là du positivisme car même s'il insiste sur l'importance de l'interprétation du texte, sur la nécessité de transformer les faits en énoncés sur les faits, l'idée que la norme était là, tapie dans l'ombre des codes, en attente du cas, l'idée d'une lex ante casum n'est pas atteinte par sa démarche. «L'objet de l'interprétation, écrit-il, c'est le texte de loi comme 'support' du sens qui est fixé en lui et dont il y va de la compréhension dans l'interprétation. L' 'interprétation' (Auslegung) c'est, si nous nous attachons à la signification des mots, la décortication (Auseinanderlegung), le déploiement et l'exposition $d u$ sens contenu dans le texte mais, pour ainsi dire, encore caché (verhüllt).» ${ }^{5}$

Le sens du texte se cachait, mais il était là. Le travail du juriste, c'est jouer à cache-cache. La norme, c'est-à-dire le sens du texte, était déjà là, il suffisait de la découvrir. Le juriste ne produit pas la norme, il l'extrait; pour reprendre une métaphore employée par l'un des principaux représentants germanistes de l'Ecole historique du droit, Georg Beseler, le juriste ne façonne pas le droit, «pas davantage que le mineur ne produit le minerai que, des profondeurs de la terre, il ramène à la surface $»^{6}$. On voit ici que l'idée d'application de la règle est liée à une certaine conception de la signification qu'on peut, après le Wittgenstein des Investigations philosophiques, qualifier par simplification de «platonicienne» ou d' «augustinienne ». La signification comme quelque chose ou quelque idée capable d'arrêter le travail d'interprétation, quelque chose ou quelque idée qui ne souffre plus la discussion, la signification comme l'idée d'un pur signifié, ce que Derrida appellerait un «signifié transcendantal $»^{7}$, qui aurait échappé à toute condition de signifiant. La norme serait ainsi le signifié transcendantal du texte de loi.

\footnotetext{
K. Larenz, Methodenlehre der Rechtswissenschaft, 6éd., Berlin e. a., Springer, 1991, p. 246. Ibid., p. 271 sq.

6 Volksrecht und Juristenerecht, cité par W. Wilhelm, Zur juristischen Methodenlehre im 19. Jahrhundert. Die Herkunft der Methode Paul Labands aus der Privatrechtswissenschaft, Frankfurt a. Main, V. Klostermann, 1958, p. 81.

7 J. Derrida, Positions, Paris, Minuit, 1972, p. 30.
} 
Cet horizon transcendantal stabilise le monde des juristes car c'est lui qui permet de garantir la possibilité de fondation des décisions en droit: il est la condition de possibilité de l'activité juridique décisionnelle conçue comme activité de fonder les décisions en droit. En même temps, en entretenant l'illusion d'une «puissance en quelque sorte nulle» (Montesquieu), le modèle «fondationnel » de l'opération juridique a aussi pour fonction de masquer le pouvoir, le pouvoir du juge ou de tout «applicateur» du droit. Il exerce ainsi une fonction politique essentielle: il conjure les craintes.

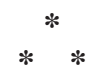

Il faut maintenant dire quelques mots sur la théorie juridique certainement la plus retentissante du $\mathrm{XX}^{\mathrm{e}}$ siècle, la Théorie Pure du Droit, le plus grand monument du positivisme juridique. Plus précisément, quelques mots de l'ouvrage de Hans Kelsen qui porte ce titre, de sa seconde édition de 1960, traduite en français en $1962^{8}$. Ce que l'on entend souligner, c'est que Kelsen, en théoricien du positivisme, mais en penseur radical et rigoureux, a mené jusqu'à son terme, a mis face à ses responsabilité, a achevé le positivisme classique en lui montrant, à partir de prémisses qu'il ne peut refuser, cela même qu'il ambitionnait de laisser dans l'ombre, ce pouvoir, ce moment politique dans le travail juridique. Mais s'il participe au et participe du travail juridique, il n'est pas en tant que tel saisissable ni maîtrisable par la science juridique qui ne peut que le montrer, comme son autre proche et menaçant, comme une «inquiétante étrangeté» dans la maison du droit.

La norme juridique, pour Kelsen, est la signification objective d'un acte de volonté. La signification «objective» est celle qui est «donnée par le droit», celle qu'un acte a «selon le droit, en droit » ${ }^{9}$. Celle-ci s'oppose à la signification «subjective» du même acte qui est celle que lui accorde son auteur et «qui est comprise par d'autres hommes $»^{10}$. Ces deux significations coïncident souvent, mais pas nécessairement. On comprend de toute façon que ce qui fait la juridicité de la norme n'est pas à chercher du côté de l'intentionnalité des acteurs, ni de celle de l'auteur, pas plus que de celle des destinataires de la normes, mais de l'instance objective appelée «droit». La norme est la signification d'un acte de volonté (et non l'acte lui-même $)^{11}$ et, en tant que cette signification est déterminée par le droit (objectif), cette norme est juridique. Il n'y a donc de norme juridique que celle qui s'insère dans le droit objectif c'est-à-dire dans un ensemble de normes données, dans un ordre juridique, un système de normes. C'est ce qui distingue l'injonction faite par le voleur au passant de lui remettre son portefeuille de l'injonction faite par l'administration fiscale de payer l'impôt: cette deuxième injonction a pour signification une norme juridique en tant que cette signification (objective) lui est donnée par le système juridique dans lequel elle s'insère (elle est l'exécution d'un

\footnotetext{
8 H. Kelsen, Théorie pure du droit, trad. fr. Ch. Eisenmann, Paris, Dalloz, 1962, rééd., Bruxelles, Paris, Bruylant, L.G.D.J., 1999.

9 Kelsen, Théorie pure du droit, op. cit., p. 11.

10 Ibid.

11 Ibid., p. 14.
} 
acte d'établissement de l'impôt pris conformément à la loi fiscale elle même adoptée conformément à la procédure législative prévue par la Constitution) ${ }^{12}$.

La science du droit ayant pour objet évident le droit, elle a donc pour objet les normes juridiques, celles-ci formant système. «La science du droit vise à comprendre son objet 'juridiquement', c'est-à-dire du point de vue du droit. Mais comprendre quelque chose juridiquement, c'est évidemment, ce ne peut être que le comprendre comme droit, autrement dit: comme norme juridique ou contenu d'une norme juridique, comme déterminé par une norme juridique ${ }^{13}$.»

Tels sont les principes essentiels, inauguraux du positivisme normativiste de Kelsen. Ils appellent quelques brèves remarques préliminaires avant d'en venir à ce qui intéresse plus spécialement les questions abordées plus haut.

D'abord une remarque très générale: qu'elle soit lue ou non de manière approfondie, la Théorie Pure du Droit tient lieu, chez les juristes, très généralement, de background théorique général. Il importe assez peu d'en étudier le détail, on sait qu'elle est là et qu'elle justifie, d'une manière où d'une autre, le positivisme juridique et donc les pratiques ordinaires des juristes contemporains. Même une théorie des plus radicales, celle que défend en France Michel Troper et qu'on a pris l'habitude d'appeler «théorie réaliste» - on y reviendra - ne sait rompre avec le kelsénisme, alors même qu'elle détruit la justification des pratiques ordinaires des juristes. Ceci pour dire simplement le poids spécifique qui revient à la Théorie pure dans le débat contemporain.

Deuxième remarque: La présentation faite plus haut ouvre évidemment sur un premier abîme. Si la juridicité d'une norme juridique lui est conférée par une (des) norme(s) juridique(s) déjà existante(s), cette (ces) dernière(s) doi(ven)t avoir elle(s)-même(s) reçu leur juridicité d'une norme juridique préexistante, et ainsi de suite à l'infini. Sans doute, cette considération explique-t-elle la structure hiérarchisée de l'ordre juridique: en tant qu'une norme $\mathrm{N}$ trouve son «fondement de validité » dans une norme N', on peut dire que N' et «supérieure » à N. Mais elle ouvre soit sur une régression à l'infini, soit sur un vide fondateur. Cela oblige Kelsen à formuler l'hypothèse d'une norme fondamentale ${ }^{14}$. C'est la norme supposée - et qui donc à ce titre n'est pas une norme positive du droit - en amont même de la constitution de l'Etat (si l'on raisonne sur l'ordre juridique de l'Etat) qui permet d'attribuer à la constitution (à l'acte positif initial) la signification objective d'une norme juridique: elle est la norme hypothétique, simplement pensée et non posée, qui permet d'attribuer à un acte constituant ou une coutume constituante la validité juridique. Mais il y a évidemment dans l'hypothèse de la norme fondamentale des difficultés propres à susciter l'inquiétude du juriste.

D'abord, lors même qu'elle est logiquement première puisque fondement ultime de validité de l'ensemble des normes d'un ordre juridique donné, supposée, elle présuppose un ordre juridique reconnu pour existant, valide, et qui a bien dû l'être pour un motif autre que celui tiré a posteriori de la norme supposée première, a priori. Une norme fondamentale se rapporte immédiatement «à une constitution déterminée, qui a été posée effectivement», et médiatement «à l'ordre

\footnotetext{
$12 \quad$ Ibid., p. $51 s q$.

13 Ibid., p. 77.

14 Ibid., p. 197sq.
} 
de contrainte qui a été posé conformément à cette constitution et qui est en gros et en général efficace» (je souligne) ${ }^{15}$. Ici, il faut bien reconnaître que l'effectivité et l'efficacité de l'ordre juridique précède et même donc conditionne la validité des normes qui le compose. La norme fondamentale supposée qui confère à l'ordre juridique sa validité présuppose elle-même l'effectivité et l'efficacité de cet ordre. Au mieux il faut y voir une violation du principe sacro-saint de la différence entre Sein et Sollen, différence «donnée à notre conscience de façon immédiate ${ }^{16}$; au pire, un cercle vicieux inextricable. Mais de cette insécurité des fondements de l'ordre juridique en tant que tel, on ne parlera pas davantage.

Plutôt que de prendre l'ordre juridique par le haut, par la pointe de la pyramide qu'il est censé former, on veut le prendre dans sa dynamique et interroger la Théorie Pure du Droit sur les modes de concrétisation du droit, la prise de décision, ce qu'on appelle ordinairement l'application.

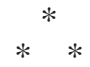

C'est sans doute une interrogation qu'il conviendrait de ne pas entreprendre. Car: «Puisque la Théorie Pure du Droit n'est qu'une connaissance du droit positif donné, mais pas une prescription pour sa production correcte, elle n'entend ni donner d'injonction s'agissant de la question de savoir comment l'on fait de bonnes lois, ni davantage donner de conseils sur les conditions dans lesquelles on peut prendre de bonnes décisions sur le fondement ou dans le cadre des lois.» ${ }^{17}$

Soit. Mais la norme, qu'elle soit individuelle ou générale, qu'elle soit la loi qui concrétise la constitution ou la décision du juge qui applique la loi, n'est norme juridique que par la signification que lui donne la norme supérieure appliquée. Si la «dynamique» de concrétisation du droit est aussi l'objet d'une science du droit conforme à la Théorie Pure du Droit, il apparaît difficile que cette Théorie fasse l'impasse sur les modes de production du droit. La norme prise dans l'ordre juridique (c'est un axiome de base de la Théorie pure) ne peut jamais y apparaître comme au repos car l'ordre juridique est avant tout et essentiellement un ordre de production du droit, un système de processus.

Si la norme juridique est la signification objective, c'est-à-dire conforme à une norme supérieure, d'un acte de volonté, il faut que la norme supérieure dispose elle-même d'une signification stable et donnée d'avance pour pouvoir garantir cette objectivité qu'elle est censé accorder aux normes inférieures. Et puisque le droit fait système, que toute norme appartenant au système est susceptible de conférer (ou refuser) sa signification juridique objective à un acte (ou un fait), cela doit valoir pour toute norme de ce système. La dynamique du droit suppose un système fixe, statique de significations.

15 Ibid., p. 201.

16 Ibid., p. 14. Une violation, car il ne s'agit pas ici d'une «corrélation» entre validité et effectivité qu'admet Kelsen (p.19) mais qui ne permet jamais de conclure de l'effectivité à la validité (pas même pour la coutume - p. 17-18) mais seulement, le cas échéant, de l'ineffectivité à l'invalidité. Ici, on procède bien de l'effectivité à la validité, la norme fondamentale faisant la médiation.

17 Kelsen, «Juristischer Formalismus und Reine Rechtslehre», cité par R. Christensen, «Strukturierende Rechtslehre», Ergänzbares Lexikon des Rechts, éd. par. N. Achterberg, Darmstadt, Neuwied, Luchterhand, 1987, 2/560, p. 2. 
La Théorie Pure du Droit s'achève par un titre VIII, de petite dimension, consacré à l'interprétation ${ }^{18}$. Kelsen y distingue l'interprétation authentique, c'est-à-dire donnée par un organe chargé d'appliquer le droit, et l'interprétation inauthentique, celle qui peut ou doit être faite par la personne qui doit suivre la règle ou par la science du droit. Le passage est essentiellement destiné à rejeter la doctrine, traditionnelle, de la solution unique: pour un cas donné, l'interprétation devrait donner une et une seule solution possible (et donc nécessaire), pour autant que cette interprétation ait été faite correctement, c'est-à-dire conformément aux méthodes destinées à cet effet. La critique de cette doctrine traditionnelle repose sur l'idée que, contrairement à ce qu'a soutenu cette doctrine, l'interprétation n'est pas, pas seulement un acte de connaissance. Plus précisément, l'interprétation par un organe juridique n'est pas un pur acte de connaissance: «L'interprétation du droit à appliquer, par une opération de connaissance, s'unit à un acte de volonté par lequel l'organe applicateur de droit fait un choix entre les possibilités révélées par l'interprétation à base de connaissance ${ }^{19}$.» $\mathrm{C}$ 'est cette adjonction d'un acte de volonté qui distingue l'interprétation authentique par un organe applicateur de droit de toute autre interprétation, en particulier l'interprétation scientifique du droit ${ }^{20}$.

L'acte de volonté est effectivement le moment de politique juridique, le moment créateur de droit dans le processus de concrétisation, le moment du pouvoir, et la théorie de l'interprétation vient au soutien d'une thèse forte de la théorie pure du droit, la thèse dite de la «formation du droit par degré » (Stufenbaulehre). On sait que cette thèse a été d'abord formulée par un autre juriste autrichien, Adolf Merkl, et que Kelsen l'a ensuite incorporée à l'édifice de la Théorie Pure du Droit ${ }^{21}$. Celle-ci prend congé de la thèse du positivisme traditionnel relativement aux sources du droit. La création du droit n'est pas la fait exclusif de certaines catégories de normes (et pour le positivisme de la loi, Gesetzespositivismus, le fait exclusif de la loi), mais un élément qui se retrouve à chaque étage du système juridique, dans chaque norme du système (abstraction faite de ses extrémités). La norme juridique possède un «double visage ${ }^{22}$ : à chaque degré du système, la norme est combinaison d'application (au sens strict) et de création du droit. A cela répond la théorie de l'interprétation authentique: en tant qu'acte de connaissance, elle reconnaît le cadre déterminant, objectif imposé par la norme supérieure (le cadre de l'application); en tant qu'acte de volonté, elle fait un libre choix parmi les possibles, cet élément subjectif étant le moment créateur du droit, le moment du pouvoir ${ }^{23}$. C'est là que se joue la dynamique du droit

\footnotetext{
18 Kelsen, Théorie pure du droit, op. cit., p. 335 à 342.

19 Ibid., p. 340.

20 Ibid.

21 Voir à ce propos, l'avant-propos à la deuxième édition de Hauptprobleme der Staatsrechtslehre, entwickelt aus der Lehre vom Rechtssatze, Tübingen, 1923, p. XV.

22 Adolf Merkl, «Das doppelte Rechtsantlitz» (1918), in H. Klecatsky, R. Marcic, H. Schambeck (éd.), Die wiener rechtstheoretische Schule, Wien e. a., Europa Verlag, Universitätsverlag Anton Pustet, 1968, p. 1091.

23 A. Merkl, art. cit., p. 1096; du même, Allgemeines Verwaltungsrecht, Wien, Berlin, Springer, 1927, p. 142.
} 
qui modifie en profondeur la vision traditionnelle: l'image du «processus ${ }^{24}$ dynamise (et dynamite) l'image traditionnelle d'un empilement figé de règles et de concepts. C'est sans doute un apport majeur de la théorie kelsénienne de transformer cette image du droit pour en faire, plutôt qu'un monument, une archive, un work in progress.

Mais c'est aussi sa difficulté principielle que d'opérer cette transformation avec des outils métaphysiques impensés, c'est-à-dire sur la base d'un cadre conceptuel qui est, au fond, le même que celui dont s'est nourri la science traditionnelle, statique et conceptualiste du droit dont il s'agit de prendre congé.

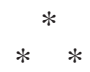

Car la Théorie Pure du Droit fonctionne sur une série de concepts, ou plus précisément d'oppositions conceptuelles métaphysiques qu'elle donne pour évidents par eux-mêmes. On l'a déjà vu s'agissant de la différence de l'être et du devoirêtre. Mais on le voit encore ici dans cette opposition de la «connaissance» et de la «volonté» qui renvoie à celle de l'«objectif» et du «subjectif». Cette dernière opposition est source d'une difficulté majeure: si la signification «objective» confère à l'acte, quelle que soit sa signification «subjective», sa juridicité et si cette signification «objective» est conférée par les normes supérieures, comment cette entrée en matière de la Théorie pure du droit parvient-elle à se concilier avec son aboutissement, sa «théorie de l'interprétation» dans laquelle la signification n'est jamais «objectivement» déterminée, mais toujours en même temps codéterminée par la subjectivité de l'interprète, par son pouvoir? Ou encore, et c'est en fait la même question formulée autrement: où est l'élément fixe (objectif) nécessairement supposé pour l'identification du système juridique dès lors que celui-ci apparaît livré au «processus » (flux des subjectivités qui l'agissent)? Ou bien encore: le droit positif, posé (gesetzt) ne repose sur rien (d'objectif). Ou bien encore, et ce serait au fond toujours la même question: à partir du moment où mon dualisme fondamental m'entraîne à séparer le juridique (objectif) du politique (subjectif), tout en les montrant tout deux à l'œuvre conjointement dans la concrétisation du droit, cette séparation l'emporte-t-elle sur la conjonction à l'œuvre, suffisamment pour permettre de croire encore à ce cadre fixe qu'on appellerait le «droit»? La contradiction ou la tension serait la suivante: le système a besoin d'un moment objectif, conçu comme moment de la détermination, du posé, du fixe que la théorie de ce système non seulement ne peut garantir, ne peut permettre de découvrir, mais plus encore: tend à remettre en cause.

En effet, en tant qu'activité de connaissance, l'interprétation scientifique du droit, par opposition à l'interprétation authentique ne peut que mettre en évidence le moment de la détermination, le moment objectif de la norme à appliquer. Dans le vocabulaire de Kelsen, la science ne peut être que description et faisant, au-delà du cercle limité de son interprétation, un acte de volonté pour fixer le contenu de la norme, non seulement elle tomberait dans le subjectif mais encore dans le pres-

24 Voir: Frantisek Weyr, «La notion de 'processus juridique' dans la théorie pure du droit », Studi filosofico-giuridici dedicati a Giorgio del Vecchio, t.2, Modène, 1931, p. 414sq. 
criptif. Seulement les méthodes d'interprétation lui sont pour ainsi dire dérobées. Comment même l'interprétation scientifique peut-elle procéder pour déterminer ne serait-ce que le cadre objectif qui doit limiter l'espace de discrétionnarité qu'il reviendra à l'autorité d'application du droit de remplir par un choix subjectif, cela n'est pas dit et cela ne peut être dit. Car il se produit quelque chose de décisif dans cette théorie, à la fin de l'ouvrage: en même temps que le mythe de la «solution unique » est évacué, et parce que les «soi-disant» méthodes d'interprétation ne peuvent donc fournir «aucun critérium sur la base duquel l'une des possibilités données dans le cadre du droit à appliquer pourrait être préférée aux autres », parce qu'il «n'y a purement et simplement aucune méthode que l'on puisse dire de droit positif qui permettrait de distinguer, entre plusieurs significations linguistiques d'une norme, une seule qui serait la vraie signification», les méthodes sont elles aussi congédiées. Elles le sont au nom d'une certaine conception de l'objectivité (et de la subjectivité, de leur opposition catégorique). Elles le sont parce qu'elles sont incapables de garantir la «vérité » de l'interprétation, la certitude de son adéquation au vrai sens de la norme. Mais il faudrait là une conclusion plus radicale, et même une conclusion double, la deuxième étant celle qui pourrait nous permettre de nous sauver d'une situation extrêmement périlleuse.

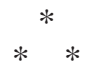

La première conclusion consiste à dire qu'à la vérité il n'y a rien de déterminé avant, dans chaque cas donné, l'intervention de l'interprète (authentique). Qu'il n'y a pas de norme avant sa signification et que celle-ci étant librement déterminée par l'interprète (le cadre «objectif », prédéterminant n'existant en vérité pas), la norme n'est pas l'objet de l'interprétation, mais bien son résultat.

Cette radicalisation est celle qu'entreprend en France la «théorie réaliste» défendue par Michel Troper. C'est l'interprète qui «insère» la norme dans le texte ${ }^{25}$; l'activité de l'interprète est absolument libre et il n'a pas à rendre compte de quelques méthodes que ce soit; la question des méthodes ne se pose pas, et s'il motive «en droit» la décision qu'il prend, l'interprète, par cette motivation ne dévoile pas le processus réel et rationnel de sa prise de décision, mais le masque ${ }^{26}$ : la motivation répond davantage à la nécessité de faire face à des contraintes de fait, des contraintes politiques (l'argumentation et la motivation est alors un simple jeu de langage politique) qu'à une obligation juridique.

Cette radicalisation opère une transmutation d'apparence étonnante: la fleur normativiste donne un fruit purement décisionniste ${ }^{27}$. Pourtant elle s'opère d'une simple correction, somme toute légitime, du texte kelsénien: l'interprétation n'est

25 M. Troper, «Le problème de l'interprétation et la théorie de la supralégalité constitutionnelle», Recueil d'études en hommage à Charles Eisenmann, Paris, Cujas, 1975, p. 142.

26 M. Troper, «La motivation des décisions constitutionnelles », La motivation des décisions de justice, Bruxelles, Bruylant, 1978, p. 301.

27 Voir aussi notre «Présentation du traducteur» in: F. Müller, Discours de la méthode juridique, op . cit., p. $7 s q$. 
plus du tout acte de connaissance, elle est purement et simplement acte de volonté ${ }^{28}$.

Cette radicalisation n'est pas assez radicale. Elle ne rompt pas avec les dualismes du kelsénisme: être/devoir-être; connaissance/volonté; subjectif/objectif. Elle reste marquée par les origines métaphysiques du kelsénisme. Mais, au fond, le nihilisme même n'a de sens que s'il maintient un certain horizon de Dieu, qui donne le contraste. C'est l'idée même de Dieu qui permet au nihiliste de jouir de son nihilisme. Ici, le «réaliste» ne jouit de son œuvre de destruction de la science du droit (qui n'a plus de légitimité, plus de méthodes, plus de sens) que parce qu'il reste sur les lieux de son crime, parce que son point de vue lui permet de contempler, dans leur cadre naturel et ordinaire, les décombres d'un droit défunt (et dans ces décombres, s'agiter encore d'innombrables juristes, à la recherche, encore et toujours, de l'arche d'alliance, qui croient, encore et toujours, pouvoir sanctifier la décision par la loi).

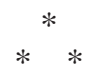

La seconde conclusion, en étant plus radicale encore, pourrait peut-être nous sauver. C'est un conseil qui invite à quitter le monde de la métaphysique, c'est-àdire à détourner le regard, et à reprendre les problèmes là où le kelsénisme nous les a laissé en friche: qu'est-ce que la concrétisation des normes? Non pas partir à la recherche inutile de la vraie méthode qui nous donnera la vraie interprétation, mais rompre radicalement avec une conception «platonicienne» de la règle, comme Wittgenstein, le second Wittgenstein, a rompu avec le prototype platonicien de la signification. Et, de là, présenter les choses autrement, dans le souci pragmatique non pas de réaliser l'irréalisable (nous donner les critères de la « vérité » des résultats de notre travail, si par vérité on entend la correspondance de ce résultat à une grandeur extérieure et préexistante à ce travail) mais de faire mieux, de ne pas désarmer, de ne pas nous satisfaire de la tentative de destruction pure et simple de ce que pendant des siècles on a appelé «jurisprudence» puis «science du droit», sans plus toutefois nous illusionner sur la capacité, les prestations, le rendement ou la puissance à l'œuvre dans cette discipline. Cette tentative, c'est celle qu'a entreprise Friedrich Müller et la Théorie Structurante du Droit ${ }^{29}$.

28 Même si, à tort, on attribue cette doctrine exclusivement volontariste de l'interprétation au Kelsen du dernier titre de la Théorie pure du droit: O. Cayla, «La chose et son contraire (et son contraire, etc.)», Les études philosophiques, n³/1999, p. 291. Ce qui permet ensuite une opération de sauvetage du Kelsen du début de la Théorie pure, l'interprétation de ce début n'étant manifestement susceptible d'aucun doute, la notion de signification qu'a alors en vue Kelsen est une notion de type illocutoire «inscrivant ainsi d'emblée l'appréhension de la juridicité dans le cadre d'une théorie pragmatique de la signification »: ibid., p. 306, note1. On aimerait un début de preuve. Le problème, c'est que tout le discours kelsénien est saturé d'une conceptualité dualiste précisément non pragmatique (et de quelle «théorie pragmatique» doit-il s'agir?).

29 En français: F. Müller, Discours de la méthode juridique, op. cit. En allemand, l'ouvrage fondamental est: Strukturierende Rechtslehre, $2^{\mathrm{e}}$ éd., Berlin, Duncker \& Humblot, 1994. C'est n'avoir pas compris la démarche de F. Müller que de la présenter comme un exemple d' «attachement à la méthodologie interprétative», comme le fait O. Cayla, art. cit., p. 293, note2. 
La norme, la norme juridique ${ }^{30}$ ne préexiste pas à son «application». Disant cela, on ne vise pas une «essence» de la norme ${ }^{31}$. On réforme bien davantage nos outils linguistiques et notre système sémantique. Si le langage est, comme le dit Wittgenstein, une «boîte à outils », la Théorie Structurante propose de changer les outils linguistiques de la science juridique en rejetant le mot d' «application » dont les connotations, on l'a dit plus haut, conduisent précisément à des représentations essentialistes, et en appelant «norme juridique » le résultat du travail (bien ou mal) fait sur les textes et les faits et qui détermine la décision d'espèce (la norme-décision). Au départ de ce travail, il y a des énoncés, des «textes de norme ${ }^{32}$, et des faits (les circonstances de l'espèce) rapportés, qui supposent d'être racontés, des «données linguistiques primaires» (textuelles) et des «données linguistiques secondaires » (la traduction linguistique des «faits »). Le travail impose la mise en relation de ces deux types de données qui doivent être progressivement élaborées: les «faits» déterminant le choix et l'interprétation des textes (élaboration du «programme normatif» de la norme), les textes déterminant le processus de sélection et de typification des faits (élaboration du «champ normatif » de la norme). La structure de la norme juridique est formée du programme normatif et du champ normatif qui ne se tiennent pas l'un à côté de l'autre sans lien (auquel cas on réintroduit un dualisme et l'on n'a rien gagné) mais sont le résultat d'une co-élaboration. La norme juridique ainsi redéfinie ne se confond donc pas avec la décision d'espèce, elle conserve un caractère général en ce sens qu'elle est itérable: le champ normatif est le résultat d'un travail de typification. Cette théorie de la «normativité concrète» est liée à une réflexion méthodique mise au point dans Discours de la méthode juridique.

On veut ici seulement insister sur ce point que la Théorie Structurante du Droit entend ne pas poser au départ, supposer la distinction qui se révèle, au bout du compte, fatale pour toute entreprise de réflexion sur les méthodes de mise en œuvre du droit, la distinction du Sein et du Sollen. On ne présuppose aucune conception épistémologique a priori quand, au contraire, comme le remarque pertinemment $\mathrm{F}$. Müller, c'est bien davantage la question de la scientificité que la question du droit qui préoccupe au premier chef Kelsen ${ }^{33}$. On dira seulement: c'est le cas qui suscite la norme, pour recentrer notre regard sur le problème de la «normativité concrète». Et si l'on ne désigne plus par le mot «norme» le texte de norme, c'est pour ne plus l'y croire cachée, en attente de son interprétation. Elle est vue alors plutôt comme

30 On n'entend pas statuer ici sur la norme en général, mais seulement formuler un énoncé sur les normes du droit, les normes qui fonctionnent dans un sous-système social déterminé en même temps qu'elles le font fonctionner.

31 Au cours de la discussion qui a suivi cet exposé, Gérard Timsit a fait valoir que la faiblesse de la théorie de Friedrich Müller pourrait venir de ce qu'il n'offre précisément aucune définition de l'essence de la norme juridique. Il est vrai que la Théorie Structurante est précisément un effort pour se débarrasser des restes d' «essentialisme» dont on croit le kelsénisme (et le réalisme) pas complètement libéré. Mais on peut avoir la faiblesse de penser que c'est là précisément ce qui fait (ou devrait faire) sa force.

32 Normtext: non pas texte de la norme (qui redirait l'assignation de la norme au texte), ni « texte normatif » (la «normativité » ne vient qu'avec le travail sur le texte qui n' « est » pas par lui-même normatif), mais seulement texte susceptible de participer à l'élaboration de normes.

33 Strukturierende Rechtslehre, op. cit., p. 24. 
le résultat d'une concrétisation. La concrétisation n'est pas dite alors pour désigner l'application ni le mode syllogistique de résolution des cas: elle désigne le mode de production de la norme qui résulte d'un travail effectué sur les textes et sur les faits. Séparer être et devoir-être, c'est s'empêcher d'emblée de pouvoir présenter les choses de cette manière. Mais, en même temps, distance est aussi prise avec le modèle herméneutique traditionnel de l'interprétation ${ }^{34}$.

Dans une telle présentation de l'opération juridique, le juriste est un travailleur du droit. Son opération est productive, travail; ouvrier du droit, il façonne, usine les normes et, de ce point de vue, du vocabulaire traditionnel, l'expression de «mise en æeuvre» sonne assez juste. Le travailleur, l'ouvrier du droit est aussi responsable de son travail. Il ne peut plus masquer son pouvoir.

La norme n'est pas ici pensée comme un autre du pouvoir, sur le mode de la séparation, ni sur celui de l'encadrement. La norme est le travail du pouvoir. Cela engage à repenser ce que c'est d'un «Etat de droit». Pour le réalisme, c'est une mythologie néfaste ${ }^{35}$. Pour la doctrine dominante, c'est le règne du droit (qui suppose la fable de l' «application»). Pour le pragmatisme de la théorie structurante, ce serait plutôt un mode particulier d'organisation du travail juridique, qui, par exemple, oblige à produire certains type de textes dans lesquels sont rapportées les étapes du travail (motivation) et leur résultat (décision). Il faudrait ici insister sur ce que Friedrich Müller appelle la «structure textuelle» (Textstruktur), la texture de l'Etat de droit ${ }^{36}$.

Une «théorie» du droit, en ce sens, ne se donne plus pour tâche d'être une «théorie de la connaissance juridique ». Sans doute cela explique-t-il que le débat soit impossible avec le kelsénisme. Son objet n'est pas le «système juridique», mais l'agir juridique. Il s'agit donc d'engager réflexion sur la production des normes et non pas ni une doctrine de la connaissance et de la description des systèmes juridique, ni une doctrine de la vérité dans l'application des lois. Si elle tient un certain discours sur les méthodes juridiques, elle ne conçoit pas la méthode comme un dispositif d'arrimage des textes à leur exacte signification, mais comme des modes d'opération juridique dans un système politique, social et culturel donné. Si elle emploie le mot de rationalité et de rationalisation, ce n'est pas au sens où il s'y engagerait un discours des fondations. La ratio ici doit être libérée du Grund. Au risque de décevoir, les notions de rationalité, de vérité, d'objectivité ne conduisent pas ici au-delà du cercle où les assignerait aussi le pragmatisme américain de William James, John Dewey ou, aujourd'hui, Richard Rorty: le monde des «pratiques sociales de justifications et de contrôles ${ }^{37}$. A la recherche névrotique de la certitude objective, à l'abandon du droit à la puissance nue de la subjectivité - car le réalisme est-il autre chose qu'une version plus moderne, qu'une version relookée du droit du plus fort -, on préfère réfléchir sur et à partir des pratiques intersubjectives.

$$
\text { * * }
$$

34 Voir: O. Jouanjan, «Nommer/Normer. Droit et langage selon la 'Théorie Structurante du Droit'», Le Temps des savoirs, $\mathrm{n}^{\circ} 1$, La Dénomination, Paris, Odile Jacob, 2000, p. 39sq.

35 Voir: M. Troper, «Le concept d'Etat de droit», Droits, n¹5, 1992, p. 51 sq.

36 Discours de la méthode juridique, op. cit., p. 184 sq.

37 R. Bodei, La philosophie au XX $X^{e}$ siècle, Paris, Flammarion, 1999, p. 244. 
On ne présentera pas le détail de la Théorie Structurante du Droit. On se bornera à indiquer seulement, sur la carte des doctrines, sa position. Et cette carte n'est pas centrée sur la Théorie Structurante. Celle-ci s'y trouve bien à la marge. La raison en est simple: elle n'offre pas la paix des braves juristes rassurés par la certitude dans laquelle ils sont que leurs pratiques leur permet d'atteindre à des propositions absolument vraies sur le droit; elle est tout autant incapable de susciter la jouissance dont se nourrissent les «caractères destructeurs », les Nérons satisfaits au spectacle des ruines fumantes de l'incendie qu'ils ont provoqué (au nom du «réalisme»). Elle est frustrante, décevante, pour certains navrante. Elle rabaisse les prétentions. Elle demande elle aussi de "perdre ce que Nietzsche appelait un 'confort métaphysique'» ${ }^{38}$. Elle brise les rêves du «conceptualisme» juridique, la croyance en un «ciel des concepts juridiques» que décrit avec humour Ihering, et qui craint tout contact avec la réalité ${ }^{39}$. On aura compris que la crainte n'est plus que ce ciel nous tombe sur la tête; il est déjà tombé et le kelsénisme y a contribué. Il est plutôt que le sol ne se dérobe sous nos pieds, avec le mythe des «fondations», des solutions juridiques «fondées en droit».

Mais si elle est ainsi prête à payer le prix fort, la Théorie Structurante du Droit a tout de même l'espoir, elle aussi, d' «obtenir en retour un sens renouvelé de la communauté», elle parie qu'il peut y avoir un gain à considérer, de façon pragmatique, que «ce qui compte, ce n'est pas notre espoir de voir les choses de manière juste, mais notre loyauté à l'égard des autres êtres humains unis contre les ténèbres » et que «notre honneur réside dans la contribution que nous apportons à des projets humains faillibles et transitoires, et non à notre obéissance à des contraintes permanentes étrangères à l'homme ${ }^{40}$.

La position de la Théorie Structurante du Droit est donc au bord de la carte, ou à l'un de ses plis. On peut évidemment craindre que le chemin qui s'esquisse là et qui part vers l'inconnu, nous invitant à décentrer notre représentation du territoire juridique, à faire glisser la carte ou à la déplier, on peut craindre que ce chemin ne soit qu'une impasse. Faire ce pari et s'engager sur ce chemin, telle est la seule alternative que nous voyons à la situation du juriste «kantien» auquel on appliquera le portrait que fait Rorty du «philosophe kantien »:

«Il existe, chez les philosophes kantiens, une profonde terreur à l'idée des risques de maladie que comporte leur métier: le philosophe qui se surmène dans les recherches qu'il consacre à notre relation avec le monde peut en effet perdre les nerfs, la raison et le monde en même temps. Cela se produit lorsqu'il se retire dans un monde d'idées ou de représentations rêvé, voire, Dieu nous aide, de textes. Afin de nous garder d'une tentation de ce genre, les philosophes kantiens nous invitent à nous souvenir que seul l'idéaliste transcendantal est un réaliste empirique. Seul celui qui comprend la relation entre représentation et représenté, et souscrit à la démarche ardue, mais rigoureusement scientifique, qui caractérise le philosophe de la connaissance du XIX et le philosophe du langage, peut être transcendantal au sens requis. Car lui seul peut se représenter la représentation

\footnotetext{
R. Rorty, Conséquences du pragmatisme, Paris, Seuil, 1993, p. 308.

39 R. Ihering, Scherz und Ernst in der Jurisprudenz, réimp. Darmstadt, Wissenschaftliche Buchgesellschaft, 1992, p. $245 s q$.

40 Rorty, op. cit., p. 308-309.
} 
elle-même d'une manière exacte. C'est seulement sous l'effet d'une telle interprétation transcendantale exacte de la représentation que le Sujet Connaissance peut entrer en contact avec l'Objet, le mot avec le monde, le scientifique avec la particule, le philosophe de la morale avec la Loi et la philosophie comme telle avec la réalité comme telle.»

On n'ajoutera simplement: et le Juriste avec la Norme.

Université Robert Schuman de Strasbourg

Institut Universitaire de France 